\title{
Prevalence of Chlamydia trachomatis, Neisseria gonorrhoeae and Mycoplasma genitalium among women attending a university clinic in Pelotas, Brazil using the Aptima Combo 2 and M. genitalium Assays
}

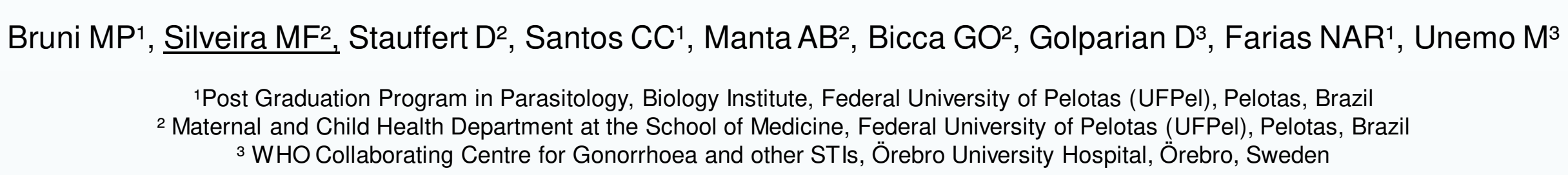

\section{Background:}

STIs caused by Chlamydia trachomatis, Neisseria gonorrhoeae and Mycoplasma genitalium are poorly diagnosed in Brazil. If these STIs are not timely detected and treated, they can result in sexual health complications such as pelvic inflammatory disease, ectopic pregnancy and tubal infertility, which severely impact particularly women in reproductive ages.

\section{Objective:}

This study aimed to investigate the prevalence of $C$. trachomatis, $N$. gonorrhoeae and $M$. genitalium infections and identify demographic, behavioral and clinical risk factors in women attending the Gynecology and Obstetrics Outpatient Clinic of the Medical School, UFPel, Pelotas, Brazil from August to December 2016.

\section{Methods:}

A total of 498 vaginal swab samples, obtained from asymptomatic and symptomatic consecutive females (one sample per female), were tested for C. trachomatis, $N$. gonorrhoeae and M. genitalium, using the US FDA-approved Aptima Combo 2 assay (Hologic) and Aptima M. genitalium assay (Hologic).

\begin{tabular}{lcc}
\hline $\begin{array}{l}\text { Prevalence of } \boldsymbol{C} \text {. trachomatis, } \boldsymbol{N} \text {. gonorrhoeae and } \boldsymbol{M} \text {. genitalium in detected vaginal } \\
\text { swab samples }(\mathbf{n}=\mathbf{4 9 8 ) .}\end{array}$ & $\mathrm{n}$ & $\%$ \\
\hline & 434 & 87.1 \\
Uninfected & 34 & 6.8 \\
C. trachomatis only & 5 & 1.0 \\
$\mathbf{N}$. gonorrhoeae only & 21 & 4.2 \\
M. genitalium only & 4 & 0.8 \\
Mixed infection & &
\end{tabular}

\section{Results:}

The mean age of the women was 36.5 years (range: 15-77 years), mean schooling was 5.5 years, and approximately $60 \%$ of patients had their own income. The prevalence of $C$. trachomatis, $N$. gonorrhoeae and $M$. genitalium was $6.8 \%$ (34/498), 1.0\% (5/498) and 4.2\% (21/498), respectively. One case of co-infection between $C$. trachomatis and M. genitalium, and three cases of co-infection between C. trachomatis and $N$. gonorrhoeae were identified. The risk factors associated with the presence of any of these STIs were young age ( $<30$ years), having no steady sexual partner, presence of co-infection, and having no income.

\section{Conclusions:}

C. trachomatis infection was the most prevalent bacterial STI (6.8\%) among women in Pelotas, Brazil. None of the examined STIs are routinely screened or tested in Brazil despite the fact that early diagnosis and treatment, including partners management, are crucial to control these STIs, to avoid future complications and to diminish the burden of those infections in the public health system. 